\title{
Avaliação das alterações hidrológicas da bacia do rio São Francisco causadas pela construção da usina hidrelétrica de Sobradinho
}

Evaluation of Hydrologic Alterations in the São Francisco River Caused by the Construction of Sobradinho Hydroelectric Plant

\author{
M. O. Santos; I. D. C. Barreto; I. M. L. Silva; T. Stosic \\ Departamento de Informártica e Estatística - DEINFO, Programa de Pós-Graduação em Biometria e Estatística \\ Aplicada - PPGBEA,Universidade Federal Rural de Pernambuco - UFRPE, 52171-900, Recife - PE, Brasil
}

\author{
daniel.carvalho.ib@gmail.com
}

(Recebido em 27 de maio de 2017; aceito em 01 de dezembro de 2017)

\begin{abstract}
Um ecossistema equilibrado é a base da vida e desenvolvimento de milhares de espécies de seres vivos. Os rios fazem um papel muito importante nesse equilíbrio. Um dos mais importantes rios do Brasil é o Rio São Francisco que contém, ao longo do seu percurso, cinco usinas hidrelétricas. As hidrelétricas são uma das fontes de energia mais baratas entre as disponíveis, por isso é a mais usada, porém a construção de hidrelétricas causa vários impactos ao meio ambiente. Para avaliar este impacto, fornecendo base para uma avaliação mais criteriosa na escolha do método de obtenção de energia e um melhor planejamento nas futuras construções de hidrelétricas, foi usado o método de Indicadores de Alteração Hidrológica (IAH) para estudar a variabilidade das características hidrológicas da vazão observada diariamente abaixo da represa de Sobradinho. Foram retirados da análise anos com chuva muito abaixo ou muito acima do normal. A partir desses dados, o método IAH gera 33 indicadores hidrológicos de relevância ecológica. Todos os indicadores de alteração hidrológica foram avaliados e os resultados mostraram que, quando comparamos o período anterior à construção da barragem com o período posterior, houve alteração hidrológica em todos os indicadores hidrológicos fornecidos pelo método de IAH.

Palavras-chave: Indicadores de Alteração Hidrológica (IAH), Sobradinho, Regulação do Rio.
\end{abstract}

A balanced ecosystem is the basis of life and responsible for the development of thousands of living species. Rivers have an important role in this balance, so there is a need to preserve its features. One of the most important rivers in Brazil is the São Francisco River, which has five hydroelectric plants along its route, including the Sobradinho hydroelectric plant (BA). Hydroelectric plants are still one of the cheapest energy sources available and the most widely used, but their construction causes various impacts on the environment. It is important to assess this impact and provide the basis for a more thorough evaluation in choosing the method of obtaining energy and better planning in future construction of hydroelectric plants. In this work, the method of Indicators of Hydrologic Alteration (IHA) is used to study the variability of hydrological characteristics of the observed daily flow below the Sobradinho dam. The IHA method generates 33 hydrological indicators of ecological relevance. All hydrologic alteration indicators were evaluated and the results showed that, when we compare the period before and after the dam construction, there were changes in all hydrological indicators provided by the IHA method.

Keywords: Indicators of Hydrologic Alteration (IHA), Sobradinho, river regulation

\section{INTRODUÇÃO}

Todos os milhares de espécies de seres vivos, na fauna e na flora, dependem de um ecossistema equilibrado para conseguir viver e se desenvolver. Para as plantas, animais e pessoas que vivem ao longo do curso de um rio, a quantidade, as variações e regularidade das águas são muito importantes. Grande parte da água usada pela humanidade vem dos rios. É usada na agricultura, pecuária, consumo, cozimento, lavagem, produção de alimentos, entre outras, por isso a importância de evitar ao máximo a poluição. Já para os animais e plantas que dependem dos rios, além da qualidade da água, qualquer outra alteração, como volume, vazão e velocidade, pode representar, direta ou indiretamente, um grande perigo para suas vidas, e, como consequência, um grande impacto na vida dos seres humanos [1]. 
Os rios também são uma importante fonte de energia elétrica. As usinas hidrelétricas visam produzir energia elétrica pelo aproveitamento do potencial hidráulico de um rio, e para isso, usam um conjunto de obras e equipamentos. Estas são ainda uma das fontes de energia mais baratas entre as disponíveis, e por isso é a mais usada. Mas, apesar do preço de produção ser baixo, para o ecossistema o preço é altíssimo. A construção de hidrelétricas causa vários impactos ao meio ambiente, como o alagamento de áreas vizinhas, o aumento do nível dos rios, podendo, inclusive, mudar o curso do rio represado. Esses fatores prejudicam a fauna e a flora da área inundada para a formação do reservatório, e também dos trechos a montante e a jusante da usina. Por esse motivo, antes da construção é necessário que se faça um estudo da viabilidade desse projeto, com o envolvimento de diversos profissionais, os quais utilizam nos seus estudos modelos matemáticos ou físicos [2].

Um dos mais importantes rios do Brasil é o Rio São Francisco. Sua nascente se localiza no estado de Minas Gerais, de onde ele segue atravessando o estado da Bahia, constituindo, em seguida, a divisa entre os estados da Bahia e Pernambuco e, por fim, representando a divisa entre os estados de Alagoas e Sergipe e seguindo para desaguar no oceano atlântico. Apresenta uma área drenada de $641000 \mathrm{~km} 2$ e comprimento de $2900 \mathrm{~km} \mathrm{[3].}$

O Rio São Francisco também proporciona para suas áreas ribeirinhas uma grande prosperidade com o uso da agricultura irrigada, como é o caso das cidades de Petrolina (PE) e Juazeiro (BA). Essa região é atualmente a maior produtora de frutas tropicais do país e também é uma das poucas regiões do mundo nas quais se obtêm duas safras de uvas por ano, o que faz a região ter um grande destaque na produção de vinhos $[4,5]$.

O Rio São Francisco contém, ao longo do seu percurso, cinco usinas hidrelétricas. Uma delas é a Usina Hidrelétrica de Sobradinho (Figura 1) que está localizada no estado da Bahia, próxima às cidades de Juazeiro (BA) e Petrolina (PE). Além da função de gerar energia elétrica, a Hidrelétrica de Sobradinho também funciona como fonte de regularização dos recursos hídricos da região. $\mathrm{O}$ reservatório é o terceiro maior lago artificial do mundo, e segundo do Brasil, e tem aproximadamente $280 \mathrm{~km}$ de comprimento, uma superfície de espelho d'água de $4.214 \mathrm{~km}^{2}$ e sua capacidade de armazenamento é de 34,1 bilhões de m3 em sua cota máxima de 392,50 m [6].

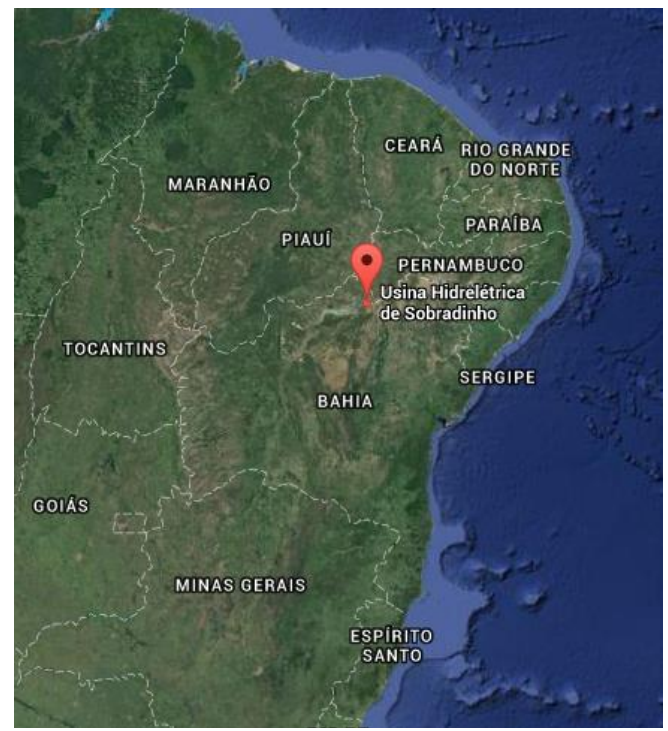

Figura 1. Localização geográfica da Usina Hidrelétrica de Sobradinho. Fonte: Google Maps

Os sistemas hidrológicos podem ser alterados por fatores naturais (variações climáticas) e pela atividade humana como construção de reservatórios e barragens, expressivas mudanças de uso de solo e construção de Sistemas de irrigação $[7,8,9]$. Entre as alterações hidrológicas que provocam degradação nos rios e ecossistemas das bacias, se destacam: as perdas de conectividade jusantemontante do rio, alteração do pulso de inundação e das relações rio-planície de inundação, alteração do regime hidrológico e alteração do regime natural da qualidade da água [8]. 
Para avaliar os efeitos de construção dos reservatórios e estabelecer os padrões de operação (liberação de água) do reservatório, é necessário quantificar as alterações hidrológicas que são ecologicamente relevantes. Vários indicadores (em total aproximadamente 170) foram propostos [10], entre quais os mais utilizados são Indicadores de Alteração Hidrológica (IAH). São 33 índices hidrológicos desenvolvidos por Richter et al. (1996) [11] que caracterizam variabilidade hidrológica inter e intra anual de acordo com cinco características de vazão: magnitude de vazões mensais, magnitude e duração de vazões extremas anuais, tempo anual das vazões extremas, frequência e duração de pulsos altos e baixos, e taxa e frequência de alteração de fluxo.

Nesse trabalho, foi usado o método de Indicadores de Alteração Hidrológica (IAH) para estudar a variabilidade das características hidrológicas da vazão observada diariamente abaixo da represa de Sobradinho, no período de 01/09/1929 até 01/09/2009. Foram calculados e comparados os indicadores IAH para os períodos anterior (1930-1972), durante e posterior (1973-2009) a construção do reservatório para avaliar o grau da alteração hidrológica.

Os estudos anteriores sobre alterações das características do regime hidrológico da bacia São Francisco causadas pelos fatores climáticos e pela atividade humana incluem os estudos de qualidade da água [12], regime de vazões [13], variação do nível do reservatório [6], impacto de evaporação líquida no escoamento [14], influência do ENOS (El Niño-Oscilação Sul) no regime hidrológico [15] e concentração de sedimentos [16]. Recentemente magnitude, duração e frequência das vazões extremos (subconjunto de indicadores IAH) foi estudado para bacia do Rio Francisco e foram comparados os indicadores nos períodos anterior a construção do primeiro reservatório (Três Marias) e depois da construção do último reservatório (Xingó).

Os resultados mostraram que os indicadores analisados foram alterados como consequência da atividade humana, com maior grau de alteração no médio e baixo São Francisco, regiões estas mais afetadas pela construção dos reservatórios [17].

Neste artigo analisamos alterações hidrológicas relacionadas a construção do reservatório Sobradinho utilizando o conjunto completo de 33 Indicadores de Alteração Hidrológica (IAH).

\section{MATERIAL E MÉTODOS}

Um dos modos de avaliar o impacto que as Usinas Hidrelétricas causam ao meio ambiente é estudando as características do rio a jusante das barragens, antes e após a construção da mesma [8]. Os dados foram obtidos no banco de dados da Agência Nacional de Águas (ANA), disponível na página http://hidroweb.ana.gov.br/ e correspondem a uma série histórica de vazões diárias, coletadas no período de 01/09/1929 até 01/09/2009, na Estação Hidrológica Juazeiro, código 48020000, que fica no município de Juazeiro, no estado da Bahia.

Os dados de precipitação, também obtidos na ANA, correspondem a uma série anual de 1929 a 2009, no município de Juazeiro (BA), de código 940016. A responsabilidade da coleta dos dados é da própria ANA.

A fim de encontrar um resultado não influenciado com condições climáticas extremas, foram retirados da análise da vazão os anos de secas e cheias, pois estes poderiam ser erroneamente entendidos como uma alteração hidrológica causada pela construção da barragem, quando na verdade seriam alterações climatológicas. Yoo (2006) [18] recomenda que sejam calculadas a média e o desvio padrão das precipitações e, em seguida, seja aplicada a seguinte regra de decisão:

- Anos com precipitação superior à média somada de $75 \%$ do desvio padrão devem ser considerados anos chuvosos e por isso devem ser retirados da análise;

- Anos com precipitação inferior à média subtraída de $75 \%$ do desvio padrão devem ser considerados anos de seca e por isso devem ser retirados da análise.

O método de Indicadores de Alteração Hidrológica (IAH) desenvolvido por Richter et al. (1996) [11] foi amplamente utilizado para avaliar a influência de barragens [7, 19, 20], reservatórios [21], uso de solo [22] e fatores climáticos [23,24] no regime hidrológico dos rios.

O IAH tem como objetivo avaliar a variação de uma série de dados hidrológicos, que devem ser coletados desde antes e até após a atividade humana geradora do suposto impacto a ser estudado. A partir desses dados, o método IAH gera 33 indicadores hidrológicos de relevância ecológica, que são divididos em cinco grupos: magnitude, duração, tempo, frequência e taxa de mudança. Quando 
comparado a outros métodos o IAH mostrou superioridade, como mostrado por Olden e Poff (2003) [25].

Para caracterizar estatisticamente a variação hidrológica, os 33 indicadores hidrológicos biologicamente relevantes gerados pelo método IAH são divididos em cinco grupos:

- Grupo 1: Magnitude.

O regime hidrológico de um rio costuma variar com o regime de chuvas passando por períodos de secas e cheias ao longo do ano. Assim, esse grupo gera 12 indicadores correspondentes às vazões médias mensais. Estes índices estão relacionados a disponibilidade de habitat para organismos aquáticos, umidade do solo para plantas e água para animais terrestres.

- Grupo 2: Magnitude e Duração de Condições Extremas Anuais

São gerados 12 indicadores que descrevem a magnitude e duração anuais dos fluxos extremos, como enchentes ou secas. Isso pode ter um grande impacto no grau de estresse ou de mortalidade no ecossistema. Os 12 indicadores são índices de número de dias com vazão nula e valores de vazão máxima e mínima (de 1 dia, 3 dias, 7 dias, 30 dias e 90 dias) além do coeficiente que mede o escoamento de base. Estes índices estão relacionados ao equilíbrio de organismos competitivos, rudes e tolerantes ao estresse, criação de novos locais para colonização de plantas, estruturação de ecossistemas aquáticos por fatores abióticos versus fatores bióticos e estruturação da morfologia do canal do rio e condições do habitat físico

- Grupo 3: Tempo Anual de Condições Extremas da Água

Corresponde à época das vazões anuais extremas e geram dois indicadores: dia da vazão máxima e dia da vazão mínima anual, segundo o calendário Juliano. Essas características podem determinar o ciclo de vida das espécies ou podem influenciar o grau de estresse ou mortalidade associado a inundações ou secas. Estes índices estão relacionados a compatibilidade com ciclos de vida de organismos, previsibilidade/evasibilidade do estresse para organismos bem como e acesso a habitats especiais durante a reprodução ou para evitar a predação.

- $\quad$ Grupo 4: Frequência e Duração de Impulsos de Alta e Baixa

Este grupo gera quatro indicadores: número de eventos de cheialseca e duração média desses eventos em cada ano. São consideradas cheias (ou impulsos de alta) períodos, dentro de um ano, em que os fluxos diários estão acima do percentual de $75 \%$ do período de pré-impacto (antes da alteração do regime do rio). É considerada seca (ou impulsos de baixa) quando os fluxos diários estão abaixo do percentual de $25 \%$ do período de pré-impacto. Porém esses percentuais podem ser modificados a depender do sistema hidrológico estudado. Estes índices estão relacionados a frequência e magnitude do estresse da umidade do solo para planos, frequência e duração do estresse anaeróbio para planos, disponibilidade de habitats de planaltos de inundação para organismos aquáticos e trocas de nutrientes e matéria orgânica entre rio e planalto de inundação.

- Grupo 5: Taxa de Frequência de Mudanças de Condições de Água

Utilizando a vazão diária, são calculados três indicadores: taxa de aumento (médias de todas as diferenças positivas entre os valores diários consecutivos), taxa de queda (médias de todas as diferenças negativas entre os valores diários consecutivos) e número de reversões. Essas taxas podem estar ligadas à capacidade de raízes de plantas de manter contato com o abastecimento de água do lençol freático. Estes índices estão relacionados ao estresse por seca sobre os planos, atração de organismos em ilhas, planícies de inundação e esforço de dessecação em organismos da borda do fluxo de baixa mobilidade (zona varial). 
Para gerar os 33 indicadores de alteração hidrológica foi usado o IAH software. Este software está disponível gratuitamente na página da internet: www.conservationgateway.org.

\section{RESULTADOS E DISCUSSÃO}

A variação anual da precipitação pode ser observada na Figura 2. A precipitação média nos anos avaliados foi $455 \mathrm{~mm}$ e o desvio padrão foi $219 \mathrm{~mm}$. Os anos em que a precipitação foi superior à média somada de $75 \%$ do desvio padrão foram considerados anos chuvosos e por isso foram retirados da análise. Já os anos com precipitação inferior à média subtraída de $75 \%$ do desvio padrão foram considerados anos de seca e por isso foram retirados da análise.

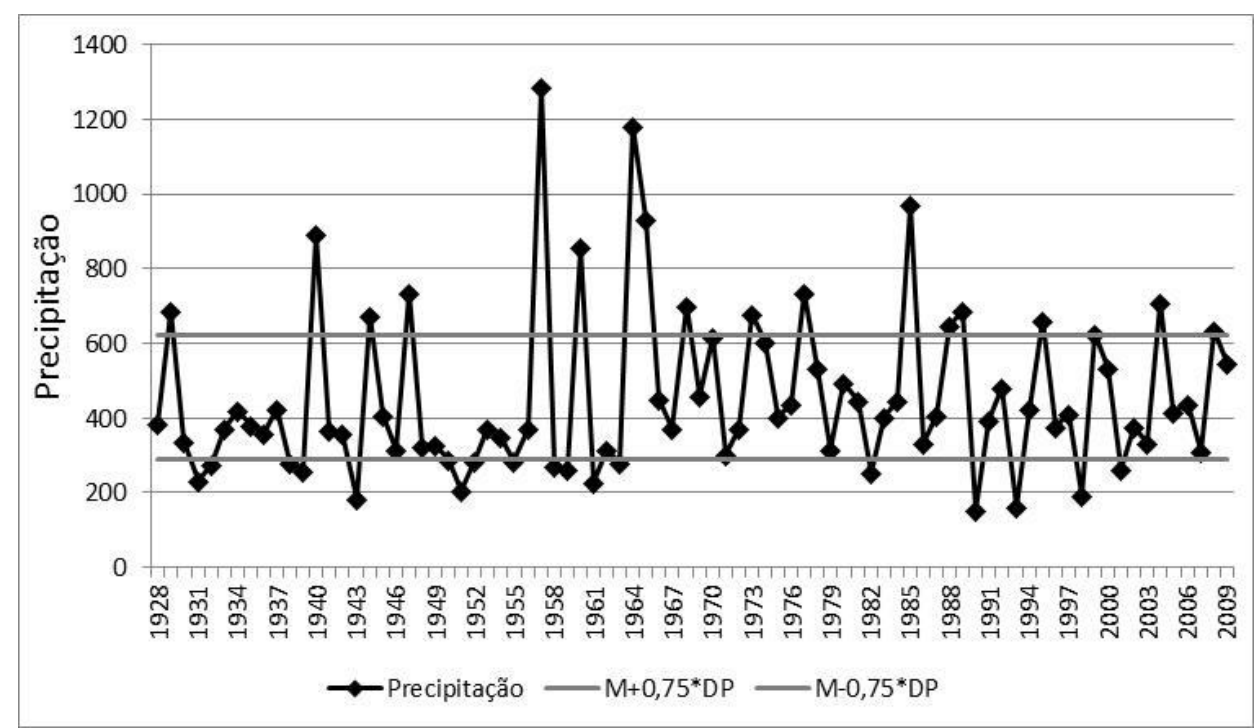

Figura 2. Precipitação anual e limites de aceitação dos anos para análise

A Figura 3 mostra o gráfico da vazão nos anos de 1929 até 2009. Os trechos onde o gráfico é descontinuado representam os anos que foram retirados da análise, como mencionado anteriormente. É possível observar que a amplitude de variação é diminuída após a construção da barragem.

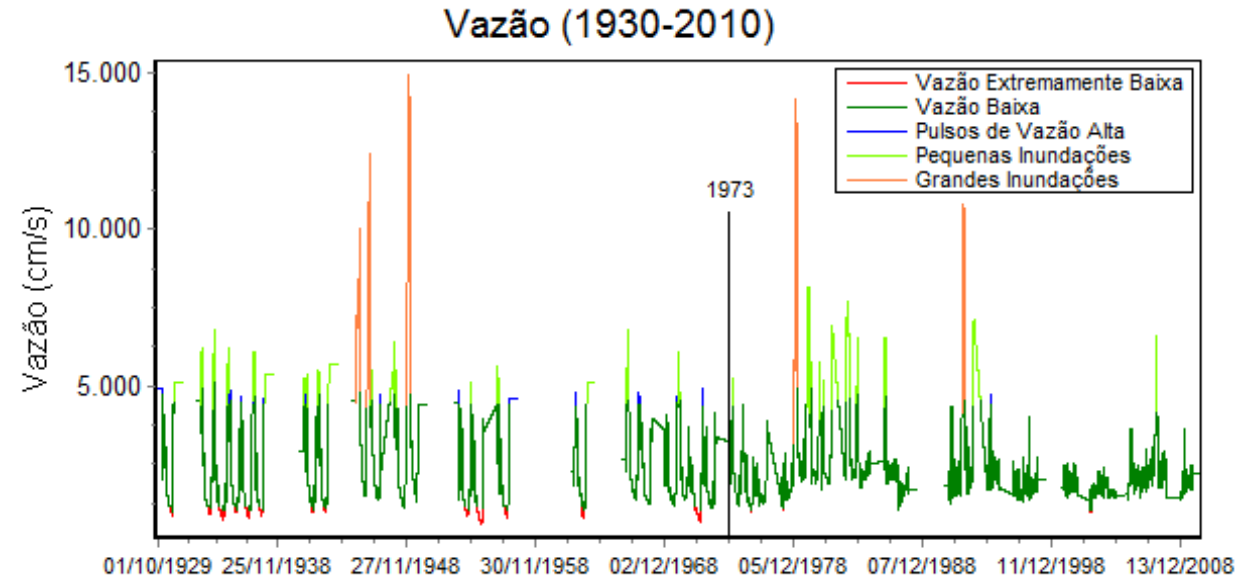

Figura 3. Vazão dos anos de 1929 até 2009 retirando-se os anos com precipitação muito alta ou muito baixa

Os resultados do Grupo 1 dos IAH (variabilidade da magnitude mensal) indicam que, na maioria dos meses, houve uma grande alteração no regime do rio quando comparados os períodos pré e pós construção da barragem de Sobradinho, como mostra a Figura 4. Observa-se que, enquanto a curva das medianas no período pré-impacto mostra um comportamento aparentemente sazonal e de grande variação, a curva do período pós-impacto se apresenta mais estável. As curvas somente se 
aproximam nos meses de novembro, maio e junho, que são os meses em que os regimes de chuva se alteram (chuvoso/seco). Também, é possível observar uma grande variação na mediana das vazões nos meses de janeiro, fevereiro e março, que costumavam ser períodos de alta vazão antes da construção da barragem. Este resultado sugere que a construção da barragem provocou maior alteração nos períodos de alta vazão do que nos períodos de baixa vazão o que sugere uma redução na biodiversidade, pois a vazão desempenha um papel importante na vida dos peixes tendo em vista que eventos como fenologia de reprodução, comportamento de desova, sobrevivência larval, padrões de crescimento e recrutamento estão diretamente ligados a vazão [26]. Além de alterações ligadas a biodiversidade, um aumento da erosão nas margens do rio pode ser observado, pois a alta vazão é responsável por levar o sedimento por todo a extensão do rio o que modela o canal no processo [27]. Como a frequência de eventos de cheia (alta vazão) ocorrem com menor incidência, observa-se um processo erosivo marcante no Baixo São Francisco [28,29].

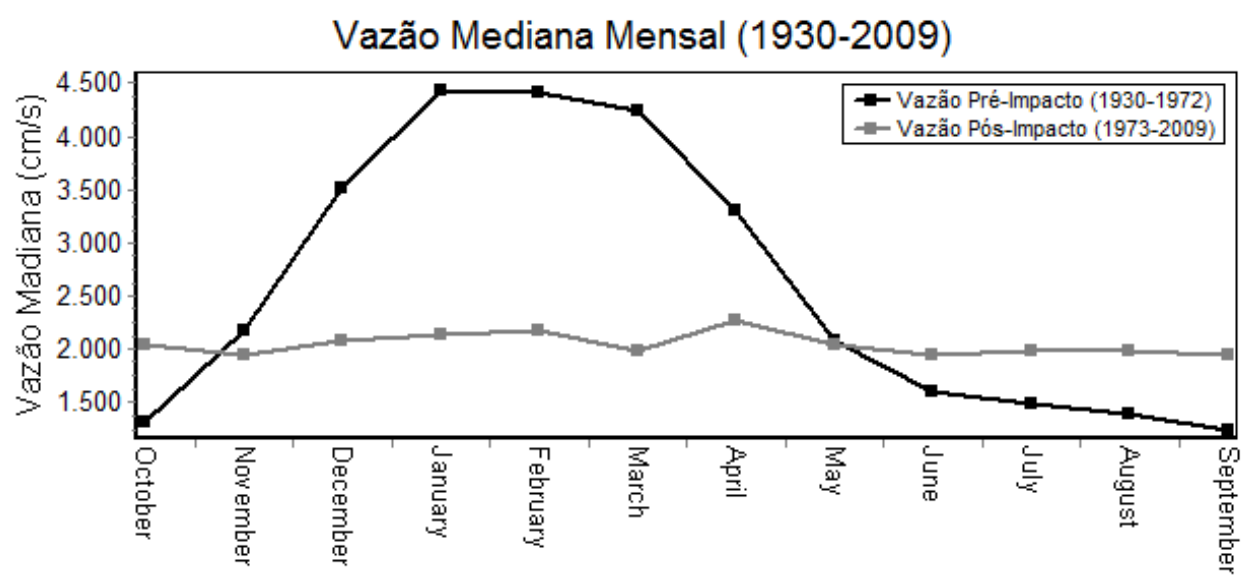

Figura 4. Medianas mensais da vazão nos períodos pré-impacto (preto) e pós-impacto (cinza)

O segundo grupo dos indicadores de alteração hidrológica revela o comportamento anual da magnitude e duração dos eventos extremos (secas e cheias). Da Figura 5 até 8 são apresentados os valores da vazão mínima e máxima anual de 1 dia e os valores da vazão mínima e máxima anual de 30 dias consecutivos (média das vazões diárias). No caso da vazão mínima de 1 dia e 30 dias consecutivos, os gráficos apresentaram o percentil 25 do período pós-impacto superior ao percentil 75 do período pré-impacto, indicando que os valores saíram completamente dos limites habituais do período pré-impacto.

Situação semelhante acontece na avaliação da vazão máxima anual de 1 dia e de 30 dias consecutivos, porém com menos intensidade de deslocamento do percentil 75. Observa-se também que, no geral, a vazão mínima anual aumentou enquanto que a vazão máxima diminuiu, confirmando o resultado obtido no grupo 1 dos indicadores de alteração hidrológica. Um resultado semelhante foi obtido por Martins et al. (2011) [13] para vazão nos trechos de baixo São Francisco após a construção do reservatório Sobradinho. Do ponto de vista da duração dos eventos extremos, os resultados sugerem que existe uma maior variação nos eventos de mínima do que nos eventos de máxima vazão.

As mudanças observadas nos grupos 1 e 2 indicam que pode haver uma redução na biodiversidade [30]. Segundo Lees et al (2016) [31] alterações na sazonalidade da vazão podem levar a extinção de espécies nos reservatórios e a jusante dos mesmos. Eventos dessa natureza foram observados em todo o planeta, contudo o único fator associado a extinção de espécies nativas mesmo que fracamente foi a construção de reservatórios pois estas atuam diretamente no grau de conectividade de subpopulações de espécies diminuindo a permeabilidade e disponibilidade de habitats entre áreas de drenagem [32]. 

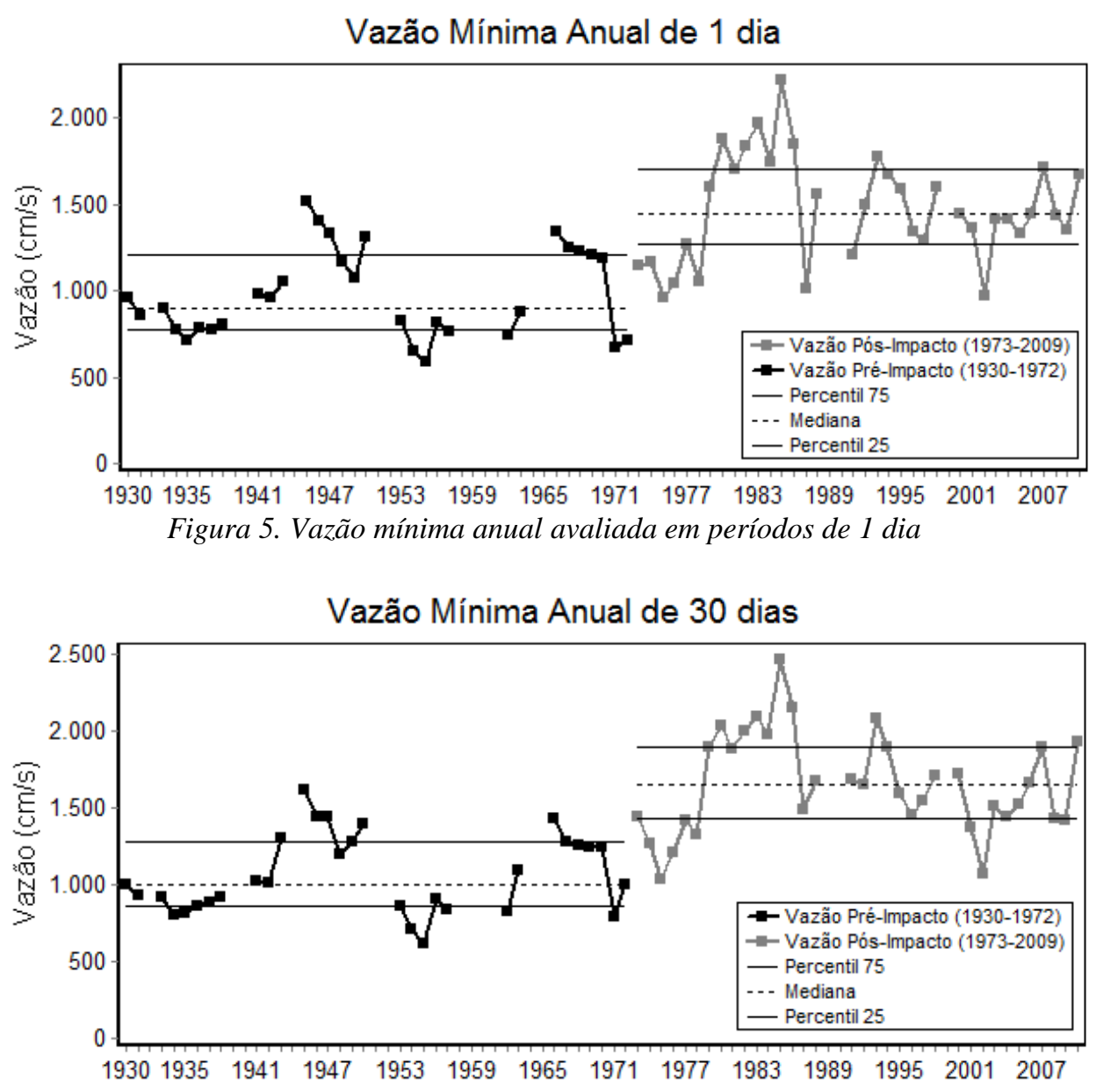

Figura 6. Vazão mínima anual avaliada em períodos de 30 dias consecutivos

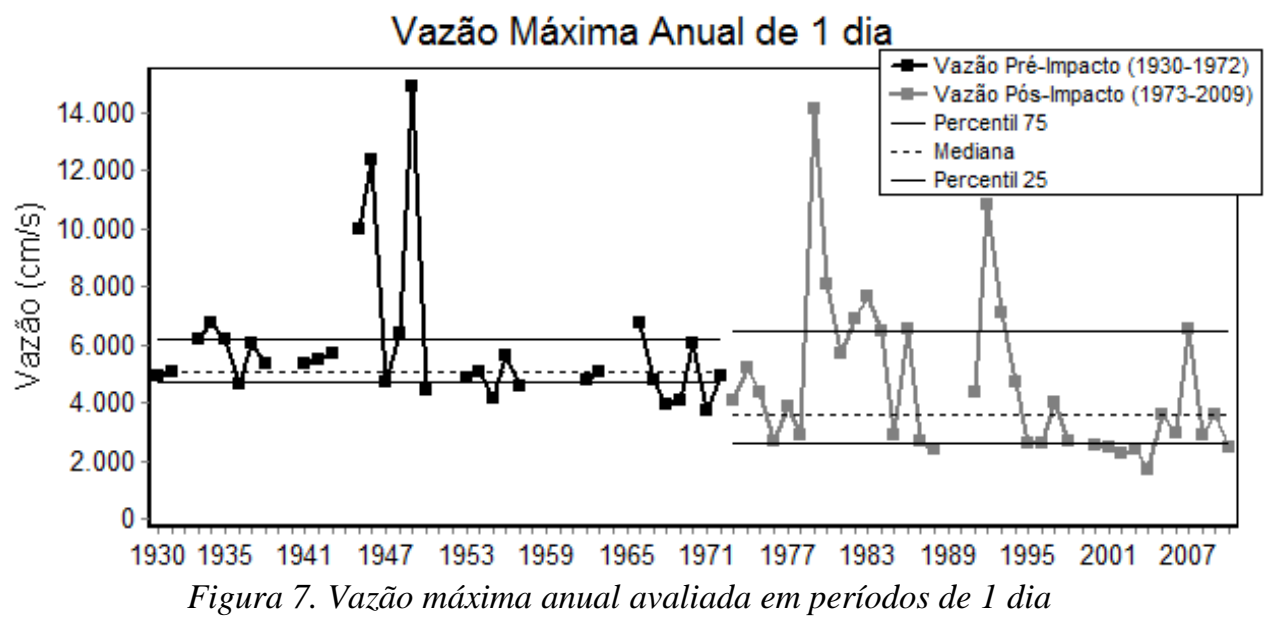




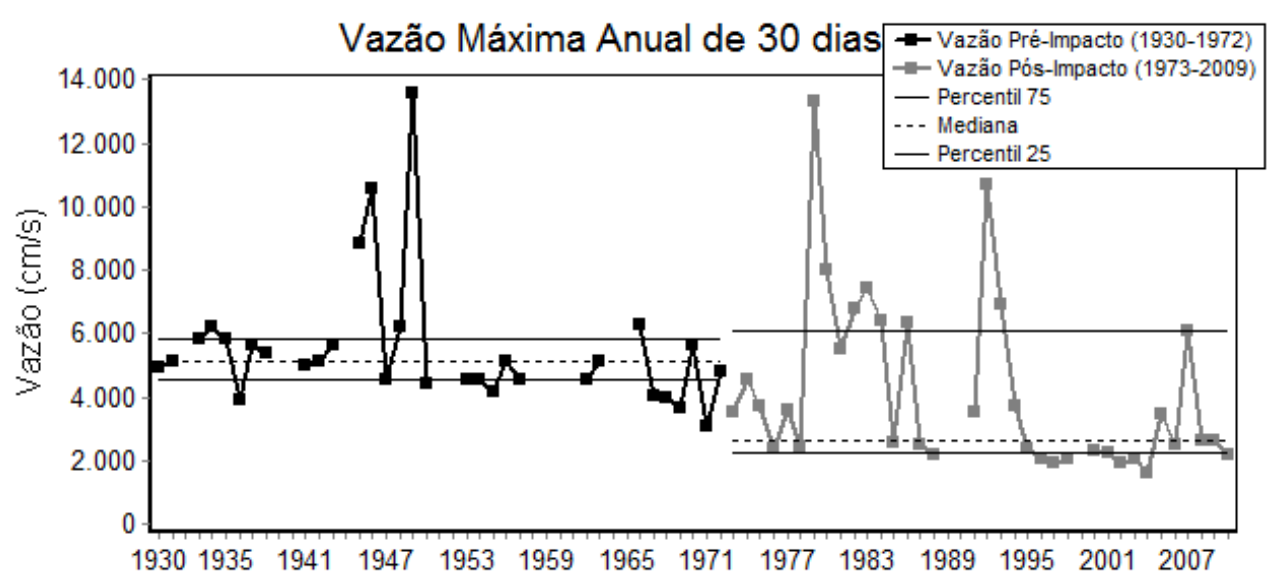

Figura 8. Vazão máxima anual avaliada em períodos de 30 dias consecutivos

O dia da vazão máxima e mínima anual, segundo o calendário Juliano, é avaliado a partir dos indicadores do Grupo 3 do IAH. A cada ano são registrados os dias de vazão máxima e mínima e depois é calculada a mediana desses dias separados em dois grupos: antes e após a construção da barragem. Na Tabela 1 podemos observar que a mediana do dia da vazão máxima no período após a construção da barragem de Sobradinho foi atrasado em 299 dias em relação ao período anterior à construção da barragem, com mudança também no coeficiente de dispersão, indicando uma precisão inferior à que se tinha antes da construção da barragem.

O dia do mínimo sofreu uma alteração semelhante, tanto nas medianas, que mostram uma antecipação de 239 dias no dia de mínimo. O coeficiente de dispersão que apresentava uma maior estabilidade $(0,07)$ antes da construção da barragem e teve um grande aumento $(0,49)$ após a construção, perdendo precisão. Essa alteração interrompe a desova de peixe com ovos pelágicos, porque a atividade de desova de muitos peixes é desencadeada pela elevação da água e pelo aumento da temperatura na primavera [33-35]. Além disso, o uso de reservatórios modifica o acesso dos peixes as zonas de desova [36] mesmo quando há escada de peixes pois o fluxo de peixes jovens ou adultos é mínima [37].

Tabela 1. Dia da vazão máxima e mínima segundo o calendário Juliano e seus coeficientes de variação

\begin{tabular}{lcccc}
\hline \multirow{2}{*}{ Parâmetros do Grupo 3 } & \multicolumn{2}{c}{ Medianas } & \multicolumn{2}{c}{ Coeficiente de Dispersão } \\
\cline { 2 - 5 } & Pré-Impacto & Pós-Impacto & Pré-Impacto & Pós-Impacto \\
\hline Dia do Mínimo & 274 & 35 & 0,07 & 0,49 \\
Dia do Máximo & 34 & 333 & 0,18 & 0,28 \\
\hline
\end{tabular}

Os pulsos de alta e baixa são avaliados pelo Grupo 4 dos indicadores IAH. As medianas das frequências de alta e baixa em cada ano são calculadas no período anterior e posterior à construção da barragem. Os resultados estão exibidos na Tabela 2, onde é possível observar que os pulsos de alta, que costumavam ser em média 1 por ano antes da construção da barragem, desapareceram após a construção da barragem, enquanto os pulsos de baixa não foram reduzidos, mantendo se 1 por ano em média.

A duração dos pulsos de alta e baixa, que, em ambos os casos, foi bastante reduzida, porém com uma redução extrema na duração dos pulsos de baixa. Assim, os resultados indicam que a construção da barragem foi importante no controle dos pulsos de alta e baixa e também na sua duração, indicando que o comportamento habitual deste trecho do rio foi bastante alterado, o que pode trazer sérias consequências para o ecossistema que depende do rio.

Estes resultados são semelhantes com os obtidos por Santos et al. [17], que encontraram a diminuição de duração de vazões baixas e da frequência das vazões altas nos trechos de baixo São Francisco depois a construção dos principais reservatórios. Essa diminuição dos pulsos de baixa e alta leva a uma menor densidade das espécies de peixe, criando uma maior predação de outras espécies e atividade extrativista humana de pesca o que ocasiona escassez do recurso [38,39] e a introdução antrópica de espécies exóticas [36,40-42]. 
Tabela 2. Frequência e duração dos pulsos de alta e baixa avaliados antes e após a construção da barragem de Sobradinho

\begin{tabular}{lcccccc}
\hline \multirow{2}{*}{ Parâmetros do Grupo 4 } & \multicolumn{2}{c}{ Medianas } & \multicolumn{2}{c}{ Coeficiente de Dispersão } & \multirow{2}{*}{ Taxa de Variação } \\
\cline { 2 - 5 } & Pré-Impacto & Pós-Impacto & Pré-Impacto & Pós-Impacto & \\
\hline Frequências dos Pulsos de Baixa & 1 & 1 & 2,00 & 4,00 & 0,00 \\
Duração dos Pulsos de Baixa & 69 & 3 & 1,59 & 6,67 & 0,96 \\
Frequências dos Pulsos de Alta & 1 & 0 & 1,00 & 0,00 & 1,00 \\
Duração dos Pulsos de Alta & 37 & 27,25 & 3,26 & 2,78 & 0,26 \\
\hline
\end{tabular}

O Grupo 5 dos indicadores avaliam a frequência de mudança das condições da água. São calculados três indicadores: taxa de aumento (médias de todas as diferenças positivas entre os valores diários consecutivos), taxa de queda (médias de todas as diferenças negativas entre os valores diários consecutivos) e número de reversões. O número de reversões está ligado à mudança de padrão de aumento ou diminuição da vazão. Assim, números pequenos de reversões ao longo de um ano indicam um comportamento persistente no aumento/diminuição da vazão. Por outro lado, números grandes de reversões indicam um comportamento anti-persistente no aumento/diminuição da vazão.

Na Tabela 3 observa-se que após a construção da barragem o aumento médio na vazão teve valor superior quando comparado ao período anterior à construção da barragem, bem como perdeu precisão no aumento médio da vazão, pois o coeficiente de dispersão aumentou de 0,47 para 0,70 . A taxa de queda se manteve praticamente estável, porém o coeficiente de dispersão quase quadruplicou, indicando uma grande perda de precisão da média estimada. Quanto ao número de reversões, observa-se que, no período após a construção da barragem a quantidade mais que sextuplicou em relação ao valor encontrado antes da construção da barragem. Observa-se também que a estimativa do quantitativo mediano de reversões se tornou menos preciso após a construção da barragem, como mostram os valores dos coeficientes de dispersão nos respectivos períodos.

Esses resultados indicam uma possível quebra do ciclo de vida biológico resultando no desaparecimento de espécies nativas e invasão de espécies exóticas [30] pois a diversidade do rio está associada ao seu ciclo hidrológico pois a vazão é fator determinante no habitat, as espécies evoluíram adaptadas ao regime natural, assim a manutenção deste regime provê sustentabilidade para estas espécies e assim quaisquer alterações no ciclo hidrológico facilitam a invasão e o sucesso de espécies exóticas [26, 45-46].

Tabela 3. Taxas de aumento e queda da vazão, e número de reversões

\begin{tabular}{lccccc}
\hline \multirow{2}{*}{ Parâmetros do Grupo 5 } & \multicolumn{3}{c}{ Medianas } & \multicolumn{2}{c}{ Coeficiente de Dispersão } \\
\cline { 2 - 5 } & Pré-Impacto & Pós-Impacto & Pré-Impacto & Pós-Impacto de Variação & 0,70 \\
\hline Taxa de Aumento & 56 & 61 & 0,47 & $-1,95$ & 0,09 \\
Taxa de Queda & -26 & $-28,5$ & $-0,50$ & 0,10 \\
Número de Reversões & 18 & 113 & 0,67 & 0,96 & 5,28 \\
\hline
\end{tabular}

\section{CONCLUSÃO}

Neste trabalho analisamos as alterações hidrológicas no rio São Francisco causadas pela construção do reservatório Sobradinho. Foi aplicado o método de Indicadores de Alteração Hidrológica (IAH) nas series temporais diárias de vazão nos períodos antes e depois da construção do reservatório. Os resultados mostraram que todas características do regime hidrológico avaliadas pelos indicadores IHA foram alterados.

Enquanto a curva das medianas das vazões diárias no período pré-impacto mostra um comportamento aparentemente sazonal e de grande variação, a curva do período pós-impacto se apresenta mais estável. Do ponto de vista da duração dos eventos extremos, os resultados sugerem que existe uma maior variação nos eventos de mínima do que nos eventos de máxima vazão.

A mediana do dia da vazão máxima no período após a construção da barragem de Sobradinho foi atrasada em 53 dias em relação ao período anterior à construção da barragem. A mediana do dia da vazão mínima sofreu uma alteração ainda maior, tanto nas medianas quanto no coeficiente de dispersão que apresentava uma maior estabilidade antes da construção da barragem.

Os resultados da análise dos pulsos de alta e baixa indicam que a construção da barragem foi importante no controle dos pulsos de alta e baixa e também na sua duração, mas é importante 
observar que o comportamento habitual deste trecho do rio foi bastante alterado, o que pode trazer sérias consequências para o ecossistema que depende do rio.

Após a construção da barragem os pulsos de alta da vazão tiveram mediana superior quando comparado ao período anterior à construção da barragem. A taxa de queda também apresentou uma variação de grande magnitude (mais que o dobro).

No período após a construção da barragem a quantidade de reversões mais que sextuplicou em relação ao valor encontrado antes da construção da barragem. O número de reversões está ligado à mudança de padrão de aumento ou diminuição da vazão.

Atualmente a vazão do rio São Francisco abaixo da barragem de Sobradinho aumenta em média com a mesma magnitude que diminui, enquanto que antes da construção da barragem o aumento mediano tinha magnitude superior à magnitude da queda.

Sendo assim, com a simplificação da estrutura física do curso natural do rio levará a uma menor deposição de sedimentos rio abaixo e consequentemente uma maior erosão na região do baixo São Francisco e a diminuição da diversidade, devido a perda da heterogeneidade de habitats tal como lagos e áreas de alagamento.

Assim, a espécies estão concentradas em uma área menor do rio levando a uma maior atividade predatória e exploratória, levando a uma possível extinção de muitas espécies. Além disso, peixes de migração podem perder suas zonas de desova devido à dificuldade de acesso, levando também a extinção destas além de um aparecimento (ou introdução) de espécies exóticas.

Com resultados deste trabalho espera-se contribuir a avaliar o impacto da construção de Usinas Hidrelétricas no regime hidrológico, proporcionando, assim, uma melhor gestão ambiental e planejamento de projetos de recuperação e gestão do ecossistema para o mais próximo possível do estado anterior.

\section{AGRADECIMENTOS}

Agradecemos a CAPES pelo apoio financeiro e a PPGBEA-DEINFO-UFRPE pelo suporte e estrutura.

\section{REFERÊNCIAS BIBLIOGRÁFICAS}

1. Acreman MC, Dunbar MJ. Defining environmental river flow requirements? A review. Hydrology and Earth System Sciences Discussions. 2004;8(5):861-76.

2. Rosenberg DM, Berkes F, Bodaly RA, Hecky RE, Kelly CA, Rudd JW. Large-scale impacts of hydroelectric development. Environmental Reviews. 1997 Mar;5(1):27-54, http://dx.doi.org/10.1139/a97-001

3. Barbosa RP. Rios brasileiros com mais de $500 \mathrm{~km}$ de extensão. Revista Brasileira de Geografia. 1962;1(126): 134.

4. Araújo GJ, Silva MM. Crescimento econômico no semiárido brasileiro: o caso do polo frutícola Petrolina/Juazeiro. Caminhos de Geografia. 2013 Jul;14(46).

5. de Lacerda MA, de Lacerda RD. O cluster da fruticultura no pólo Petrolina/Juazeiro. Revista de Biologia e Ciências da Terra. 2004;4(1):0.

6. de Fátima Correia M, da Silva Dias MA. Variação do Nível do Reservatório de Sobradinho e seu Impacto Sobre o Clima da Região. Revista Brasileira de Recursos Hidrícos. 2003 Jan/Mar;8(1):157:68.

7. Magilligan FJ, Nislow KH. Changes in hydrologic regime by dams. Geomorphology. 2005 Oct;71(1):6178. https://doi.org/10.1016/j.geomorph.2004.08.017

8. Rosenberg DM, McCully P, Pringle CM. Global-scale environmental effects of hydrological alterations: introduction. BioScience. 2000 Sep;50(9):746-51.

9. Serpa D, Nunes JP, Santos J, Sampaio E, Jacinto R, Veiga S, Lima JC, Moreira M, Corte-Real J, Keizer JJ, Abrantes N. Impacts of climate and land use changes on the hydrological and erosion processes of two contrasting Mediterranean catchments. Science of the Total Environment. 2015 Dec;15(538):64-77. https://doi.org/10.1016/j.scitotenv.2015.08.033

10. Gao Y, Vogel RM, Kroll CN, Poff NL, Olden JD. Development of representative indicators of hydrologic alteration. Journal of Hydrology. 2009 Jul;374(1):136-47, http://dx.doi.org/10.1002/rra.1155 
11. Richter BD, Baumgartner JV, Powell J, Braun DP. A method for assessing hydrologic alteration within ecosystems. Conservation biology. 1996 Aug;10(4):1163-74, http://dx.doi.org/10.1046/j.15231739.1996.10041163.x

12. da Silva DF, Galvíncio JD, de Carvalho Almeida HR. Variabilidade da qualidade de água na bacia hidrográfica do Rio São Francisco e atividades antrópicas relacionadas. Qualitas Revista Eletrônica. 2010 Aug;9(3), http://dx.doi.org/10.18391/qualitas.v9i3.687

13. Martins DMF, Chagas RM, Neto JD, Júnior AV. Impactos da construção da usina hidrelétrica de Sobradinho no regime de vazões no Baixo São Francisco. Revista Brasileira de Engenharia Agrícola e Ambiental. 2011 Oct;15(10):1054-62.

14. Pereira SB, Pruski FF, Silva DD, Ramos MM. Evaporação líquida no lago de Sobradinho e impactos no escoamento devido à construção do reservatório. Revista Brasileira de Engenharia Agrícola e Ambiental. 2009;13(3):346-52.

15. de Sousa Gurjão CD, de Fatima Correia M, Chaves Filho JB, da Silva Aragão MR. Influência do Enos (El Niño-Oscilação Sul) no Regime Hidrológico do Rio São Francisco: uma Análise em Regiões com Fortes Pressões Antrópicas (Influence of Enso (El Niño-Southern Oscillation) in the Hydrological Regime São Francisco River: an Analysis...). Revista Brasileira de Geografia Física. 2012 Nov;5(4):774-90.

16. Medeiros PR, Knoppers B, de Souza WF, Oliveira EN. Aporte de sedimentos em suspensão no baixo rio São Francisco (SE/AL), em diferentes condições hidrológicas. Brazilian Journal of Aquatic Science and Technology. 2011 Jul;15(1):42-53, http://dx.doi.org/10.14210/bjast.v15n1.p42-53

17. Santos HD, dos Santos Pompeu P, Kenji DO. Changes in the flood regime of São Francisco River (Brazil) from 1940 to 2006. Regional Environmental Change. 2012 Mar;12(1):123-32, http://dx.doi.org/10.1007/s10113-011-0240-y

18. Yoo C. Long term analysis of wet and dry years in Seoul, Korea. Journal of Hydrology. 2006 Mar;318(1):24-36, http://dx.doi.org/10.1016/j.jhydrol.2005.06.002

19. Costigan KH, Daniels MD. Damming the prairie: human alteration of Great Plains river regimes. Journal of Hydrology. 2012 Jun;444:90-9, https://doi.org/10.1016/j.jhydrol.2012.04.008

20. Yang T, Zhang Q, Chen YD, Tao X, Xu CY, Chen X. A spatial assessment of hydrologic alteration caused by dam construction in the middle and lower Yellow River, China. Hydrological processes. 2008 Aug;22(18):3829-43, http://dx.doi.org/10.1002/hyp.6993

21. Zhang Q, Xiao M, Liu CL, Singh VP. Reservoir-induced hydrological alterations and environmental flow variation in the East River, the Pearl River basin, China. Stochastic environmental research and risk assessment. 2014 Dec;28(8):2119-31, http://dx.doi.org/10.1007/s00477-014-0893-4

22. Pyron M, Neumann K. Hydrologic alterations in the Wabash River watershed, USA. River Research and Applications. 2008 Oct;24(8):1175-84, http://dx.doi.org/10.1002/rra.1155

23. Kim BS, Kim BK, Kwon HH. Assessment of the impact of climate change on the flow regime of the Han River basin using indicators of hydrologic alteration. Hydrological Processes. 2011 Feb;25(5):691-704, http://dx.doi.org/10.1002/hyp.7856

24. Dyer F, ElSawah S, Croke B, Griffiths R, Harrison E, Lucena-Moya P, Jakeman A. The effects of climate change on ecologically-relevant flow regime and water quality attributes. Stochastic Environmental Research and Risk Assessment. 2014 Jan;28(1):67-82, http://dx.doi.org/10.1007/s00477-013-0744-8

25. Olden JD, Poff NL. Redundancy and the choice of hydrologic indices for characterizing streamflow regimes. River Research and Applications. 2003 Mar;19(2):101-21, http://dx.doi.org/10.1002/rra.700

26. Bunn SE, Arthington AH. Basic principles and ecological consequences of altered flow regimes for aquatic biodiversity. Environmental management. 2002 Oct;30(4):492-507.

27. Richter BD, Richter HE. Prescribing flood regimes to sustain riparian ecosystems along meandering rivers. Conservation Biology. 2000 Oct;14(5):1467-78.

28. Holanda FS, Santos LG, Santos CM, Casado AP, Pedrotti A, Ribeiro GT. Riparian vegetation affected by bank erosion in the lower São Francisco River, Northeastern Brazil. Revista Árvore. 2005 Apr;29(2):32736.

29. Rocha IP, Holanda FS, Araújo Filho RN, Casado AP, Bandeira AA. Meteorological and hydrological variables on occurrence of riverbank erosion along lower São Francisco river. Revista Brasileira de Engenharia Agrícola e Ambiental. 2013 Feb;17(2):137-44.

30. Lin K, Lin Y, Xu Y, Chen X, Chen L, Singh VP. Inter-and intra-annual environmental flow alteration and its implication in the Pearl River Delta, South China. Journal of Hydro-environment Research. 2017 Jun;15:27-40.

31. Lees AC, Peres CA, Fearnside PM, Schneider M, Zuanon JA. Hydropower and the future of Amazonian biodiversity. Biodiversity and conservation. 2016 Mar;25(3):451-66.

32. Dias MS, Tedesco PA, Hugueny B, Jézéquel C, Beauchard O, Brosse S, Oberdorff T. Anthropogenic stressors and riverine fish extinctions. Ecological Indicators. 2017 Aug;79:37-46. 
33. Lian Y, You J, Sparks R, Demissie M. Impact of human activities to hydrologic alterations on the Illinois River. J. Hydrol. Eng. 2012;17:537-546.

34. Coimbra MR, Lima AP, Oliveira KK, Severi W. Microsatellite assessment of the genetic diversity in indigenous populations of curimba (Prochilodus argenteus) in the São Francisco river (Brazil). Conservation Genetics. 2017;1-1.

35. Normando FT, Santiago KB, Gomes MV, Rizzo E, Bazzoli N. Impact of the Três Marias dam on the reproduction of the forage fish Astyanax bimaculatus and A. fasciatus from the São Francisco River, downstream from the dam, southeastern Brazil. Environmental biology of fishes. 2014 Mar;97(3):30919.

36. Silva PS, Makrakis MC, Miranda LE, Makrakis S, Assumpção L, Paula S, Dias JH, Marques H. Importance of reservoir tributaries to spawning of migratory fish in the upper Paraná River. River research and applications. 2015 Mar;31(3):313-22.

37. Pelicice FM, Pompeu PS, Agostinho AA. Large reservoirs as ecological barriers to downstream movements of Neotropical migratory fish. Fish and Fisheries. 2015 Dec;16(4):697-715.

38. Endo W, Peres CA, Haugaasen T. Flood pulse dynamics affects exploitation of both aquatic and terrestrial prey by Amazonian floodplain settlements. Biological Conservation. 2016 Sep;201:129-36.

39. Birnie-Gauvin K, Larsen MH, Nielsen J, Aarestrup K. 30 years of data reveal dramatic increase in abundance of brown trout following the removal of a small hydrodam. Journal of Environmental Management. 2017 Dec;204:467-71.

40. Zeng L, Zhou L, Guo DL, Fu DH, Xu P, Zeng S, Tang QD, Chen AL, Chen FQ, Luo Y, Li GF. Ecological effects of dams, alien fish, and physiochemical environmental factors on homogeneity/heterogeneity of fish community in four tributaries of the Pearl River in China. Ecology and evolution. 2017 Mar 1.

41. Barbosa NP, Silva FA, de Oliveira MD, dos Santos Neto MA, de Carvalho MD, Cardoso AV. Limnoperna fortunei (Dunker, 1857)(Mollusca, Bivalvia, Mytilidae): first record in the São Francisco River basin, Brazil. Check List. 2016 Feb;12(1):1846.

42. Santos U, Silva PC, Barros LC, Dergam JA. Fish fauna of the Pandeiros River, a region of environmental protection for fish species in Minas Gerais state, Brazil. Check List. 2015 Jan;11(1):1507.

43. da Silva TC, Ramos MA, Schwarz ML, Alvarez IA, Kill LH, de Albuquerque UP. Local representations of change and conservation of the riparian forests along the São Francisco River (Northeast Brazil). Forest Policy and Economics. 2014 Aug;45:1-2.

44. Lin K, Lian Y, Chen X, Lu F. Changes in runoffs and eco-flows in the Dongjiang River of the Pearl River Basin, China. Front. Earth Sci. 2014;8(4);547-557

45. Chapin Iii FS, Zavaleta ES, Eviner VT, Naylor RL. Consequences of changing biodiversity. Nature. 2000 May;405(6783):234.

46. Assis DA, Dias-Filho VA, Magalhães AL, Brito MF. Establishment of the non-native fish Metynnis lippincottianus (Cope 1870) (Characiformes: Serrasalmidae) in lower São Francisco River, northeastern Brazil. Studies on Neotropical Fauna and Environment. 2017 Sep;52(3):228-38. 